\title{
Inverse Kinematics of Gel Robots made of Electro-Active Polymer Gel
}

\author{
Mihoko OTAKE ${ }^{\dagger} \dagger$ Yoshiharu KAGAMI $\ddagger$ \\ Yasuo $\mathrm{KUNIYOSHI}^{\dagger}$ Masayuki INABA ${ }^{\dagger}$ Hirochika INOUE $^{\dagger}$ \\ $\dagger$ Dept. of Mechano-Informatics, \\ University of Tokyo, \\ 7-3-1 Hongo, Bunkyo-Ku, Tokyo, \\ 113-8656, JAPAN \\ $\ddagger$ Complex System Engineering, \\ Hokkaido University, \\ N13-W8, Kita-ku, Sapporo, \\ 060-8628, JAPAN
}

\begin{abstract}
This paper proposes an inverse kinematic model for deformable robots made entirely of electro-active polymer gel. The required method is to control higher degrees of freedom than numbers of input. We have been proposed a kinematic and dynamic model of electro-active polymer system and derived a variety of motions of gel robots by applying spatially varying electric fields. However, inverse kinematic model and the method of applying time alternating electric fields have not been investigated. We challenge the tip control of gel manipulator by applying spatially uniform but time varying electric field. We show the procedure to control tip position of a gel manipulator by dynamically and slightly changing its whole configuration. Our work will be the first step towards the shape control of gel robots.
\end{abstract}

\section{Introduction}

Electroactive polymers are promising materials for creating deformable robots and other emerging kinds of mechanisms. For example, three dimensional displays which deform into arbitrary shapes may be realized provided we can devise methods to control the shape of these materials. Elastic manipulators for plastic surgery, and power assist suits that support the movement of human bodies are additional future applications. Biomimetic robots, such as mollusck-shaped robots, include applications for amusement and entertainment.

The current problems of electroactive polymers (EAP) are strength, safety and operating time. The performance of EAPs has been improving recently. One class of materials, electrostrictive polymers, was reported to generate strain higher than one hundred percent[1]. Micro cell manipulators[2], catheters[3], and touch displays[4] were developed experimentally. Starfish-shaped robots that

\footnotetext{
${ }^{1}$ E-mail: mike@jsk.t.u-tokyo.ac.jp
}

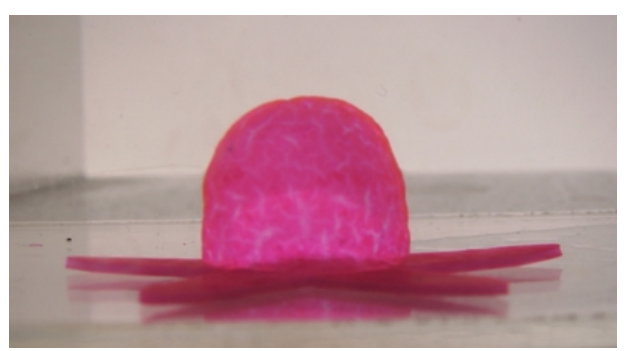

Figure 1: Prototype of octopus-shaped gel robot

turn over were also prototyped[5].

The essential theories and technologies for these applications are design and control methods that bring out the capability of the materials. Our approach to discover these methods is through prototyping a series of robots made of EAP gels, "gel robots". Initially, we proposed a shape design method for gel robots [6]. Based on the experiments, we proposed a kinematic model[7] and dynamic model[8] that describes both active and passive deformations of the gel. We conducted experiments using a spatially varying electric field generated by electrodes, and derived methods for calculating the electric field. By combining the kinematic model of the gel and the electric field model, we have generated dynamic motions [5].

However, we have not yet controlled the shape of these gel robots. The purpose of this study is to propose an inverse kinematics model which could ultimately be used to control the shape of the gel robot such as tentacle control of octopus-shaped gel robots which we prototyped (Figure 1). We focus on tip (end-effector) position control of a gel manipulator as a first step(Figure 2). This will form a foundation for shape control of gel robots. One of the primary difficulties is that the number of degrees of freedom is larger than the number of inputs, since deformable materials have conceptually infinite degrees of freedom. One approach we have used previously applies spatially 


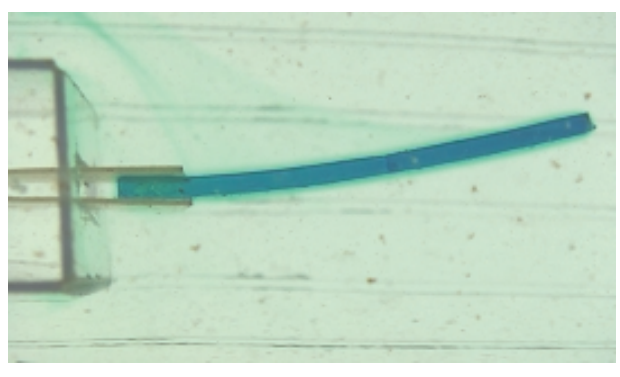

Figure 2: Gel manipulator

varying electric fields generated by multiple electrodes. Another approach, which we propose in this paper, is to apply alternating electric fields. To simplify the problem, we apply alternating but spatially uniform electric fields generated by parallel electrodes. We demonstrate the application of this method to move the tip of a gel manipulator to a desired position.

\section{Kinematic model based on mechanism}

\subsection{Deformation mechanism based on electro- chemical reaction}

We selected a typical electro-active polymer gel, poly (2-acrylamido-2-methylpropane sulphonic acid) gel (PAMPS gel) [9] [10] and its co-polymer gel from among the variety of electro-active polymers because its ability to undergo large deformations although the response speed is not so fast. The gel bends toward anode side in a surfactant solution when an electric field is applied.

The deformation of gels is a result of surface shrinking caused by the binding reaction of the surfactant molecules with the polymer network[11]. For the purposes of theoretical analysis, the binding reaction can be characterized by two processes: an adsorption process and a propagation process (see Figure 3).

Adsorption Process: electrostatic salt formation between the surfactant molecules and the oppositelycharged sulfonates of the gel.

Propagation Process: hydrophobic interaction between the bound surfactant, which stabilizes the aggregate in such a way as to settle adjacent to the already occupied site along the polymer chain.

\subsection{Kinematic model}

Based on this theory and our measurements, we proposed the following kinematic model to control shape of gels.

If we simply consider the gel as an articulated linkage made of polymer chains in two dimensional space, the gel

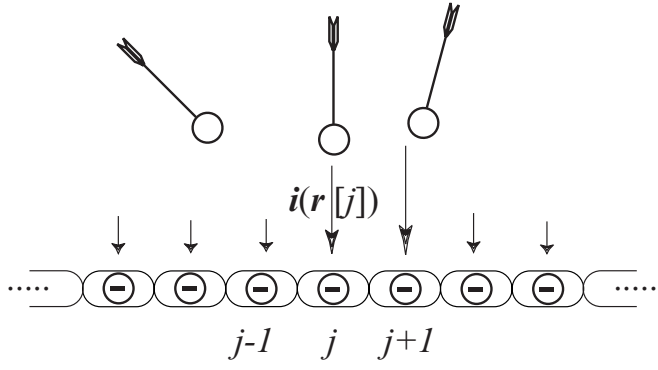

(a) Adsorption Process

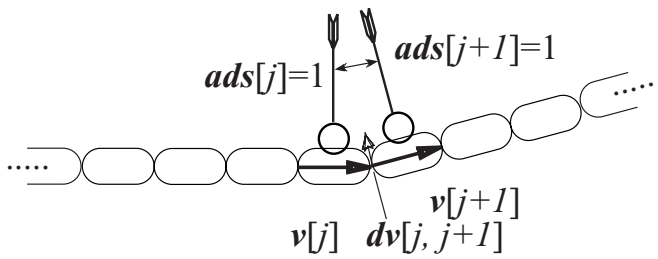

(b) Propagation Process

Figure 3: Kinematic model of electro-active polymer gel

is a collection of four components:

$$
\boldsymbol{g e l}=[\boldsymbol{r}, \boldsymbol{v}, \boldsymbol{h}, \boldsymbol{a d s}]
$$

where the jth link is formulated using the position vector $\boldsymbol{r}[j]$, orientation vector $\boldsymbol{v}[j]$, thickness $h[j]$, adsorption state parameter $\boldsymbol{a d s}[j]$. The adsorption state parameter indicates whether the link is adsorbed by molecules or not. Then the adsorption rate at link $\mathrm{j}$ is expressed as:

$$
\begin{gathered}
\boldsymbol{v a d s}=-p_{\text {ele }}\left(\boldsymbol{v}_{\perp}[j] \cdot \boldsymbol{i}(\boldsymbol{r}[j])\right)+p_{\text {ads }} \boldsymbol{a d s}[j], \\
\boldsymbol{a d s}[j]= \begin{cases}0 & (\boldsymbol{v a d s}<0) \\
\boldsymbol{v a d s} & (0 \leq \boldsymbol{v a d s} \leq 1) \\
1 & (\boldsymbol{v a d s}>1),\end{cases}
\end{gathered}
$$

with current density $\boldsymbol{i}$ on the surface of the gel $\boldsymbol{r}$ (Figure3(b)) and a vector perpendicular to the link, $\boldsymbol{v}_{\perp}[j]$. This approximately represents the adsorption process: the electrical and chemical interaction. $p_{\text {ele }}$ and $p_{a d s}$ are effect parameters of the electric field and the previous state of the adsorption. If $p_{\text {ele }}$ is large, the electric field takes large effects on the adsorption of the molecule. Once the surfactant molecule adsorbed, it takes a long time to desorb without reversing the direction of the electric field. We express this phenomenon by setting $p_{a d s}$ nearly equals to 1 . We can obtain the adsorption rate approximately by observing the joint angle of each link with a coefficient parameter $p_{d v}$ :

$$
\boldsymbol{v}[j]=\boldsymbol{v}[j-1]+\boldsymbol{d} \boldsymbol{v}[j-1, j]
$$

$$
\boldsymbol{d} \boldsymbol{v}[j-1, j]=\frac{2 p_{d v}}{h[j-1]+h[j]}(\boldsymbol{a d} \boldsymbol{s}[j-1]+\boldsymbol{a d} \boldsymbol{s}[j]) .
$$




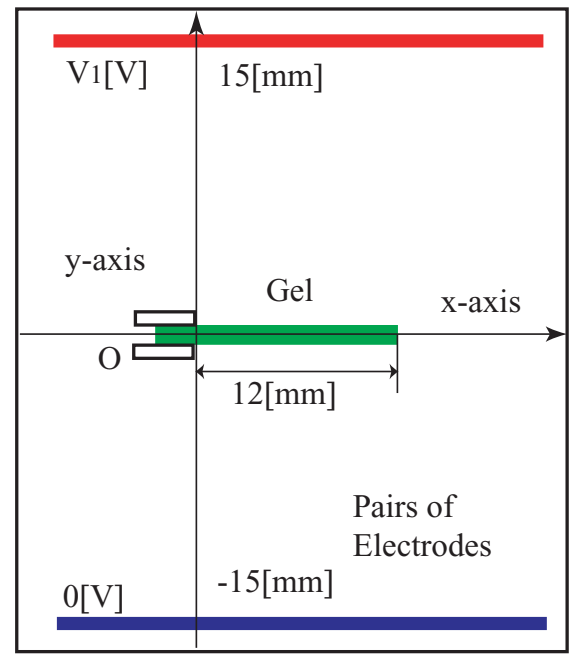

Figure 4: Structure of the gel and electric field setup

\subsection{Simulator based on the model}

We implemented a simulator based on the model. We explain our control method with simulation results. The simulator setup is shown in Figure 4. The length of the gel manipulator is $12[\mathrm{~mm}]$ and a pairs of electrodes is placed with $30[\mathrm{~mm}]$ spacing which generates spatially uniform electric fields. Draw a horizontal line (x-axis) from the root of the gel, a vertical line (y-axis) from the tip of the gel, intersecting at the point $\mathrm{O}$ (the origin). The electrodes are placed at $\mathrm{y}=15[\mathrm{~mm}]$ and $-15[\mathrm{~mm}]$. The length of the link is $\left|\boldsymbol{v}_{\perp}[j]\right|=1[\mathrm{~mm}]$, time step is $\Delta \mathrm{t}=1[\mathrm{~s}]$. Although we can apply voltage continuously, we apply discrete voltage to make the discussion simple. We apply $5[\mathrm{~V}]$ to move the tip to positive direction along y-axis, $-5[\mathrm{~V}]$ to move it toward negative direction, and $0[\mathrm{~V}]$ to stop it.

\section{Inverse kinematics of the gel}

\subsection{Tip position control}

The difference between the traditional manipulator and gel manipulator is whether we can control joint angle directly or not. The conceptual joints of the gel manipulator are coupled and calculated with equation 2 and equation 5 . Because of the above mechanism, we propose a method to generate input discrete voltage array which directly realizes desired tip position of the gel.

In general, the gel manipulator swings repeatedly if the polarity of an electric field is altered repeatedly. If the amplitude of the swinging motion is symmetric and stable, the tip of the gel moves on the same path. The tip path of the gel manipulator swings between $\mathrm{y}=6[\mathrm{~mm}]$ and $-6[\mathrm{~mm}]$, and shape at $\mathrm{t}=0,15,30,45[\mathrm{~s}]$, are shown in Figure 5 . The applied voltage and the y-coordinate of the tip is

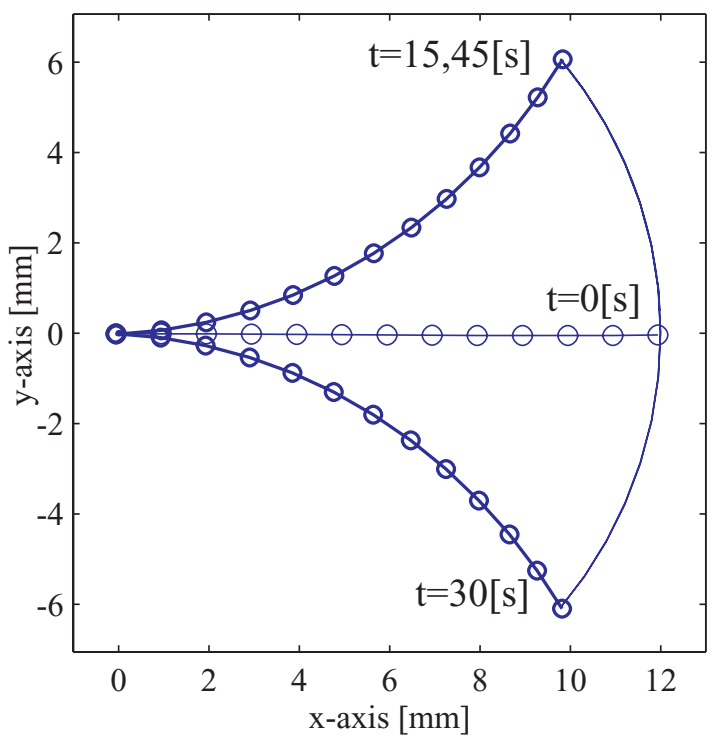

Figure 5: Tip path and final shape of gel manipulator: amplitude of $6 \mathrm{~mm}$

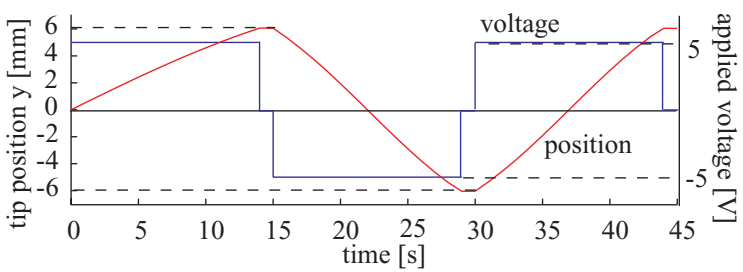

Figure 6: Applied voltage and tip position of y

shown in Figure 6 . The polarity of the voltage altered when the tip reached $\mathrm{y}=6[\mathrm{~mm}]$ and $-6[\mathrm{~mm}]$. In this paper, we show the method to move the tip of the gel from the path to the desired position. Controlling either $\mathrm{x}$ or $\mathrm{y}$-coordinate is relatively easy. We need to apply the electric field until the $\mathrm{x}$ or $\mathrm{y}$-coordinate of the tip reach the desired position. However, the coupled deformation of the gel make it difficult to control both $\mathrm{x}$ and $\mathrm{y}$-coordinate at the same time. To change the $\mathrm{x}$ and $\mathrm{y}$-coordinate of the tip independently, we designed a procedure based on mechanism. Our strategy is to utilize the nonlinearity of equation 2. The final position of the tip depends on the path of each link, because the joint angle are the function of position $\boldsymbol{r}[\mathrm{j}]$ and orientation $\left|\boldsymbol{v}_{\perp}[\mathrm{j}]\right|$. We try to change the path of each link by applying timely alternating electric fields. The deformation response of the real gel varies to the same input signal because the properties of the gels are not uniform. To reduce the effect of this scatter, we monitor the tip position and switch the polarity of input electric field based on its position. 


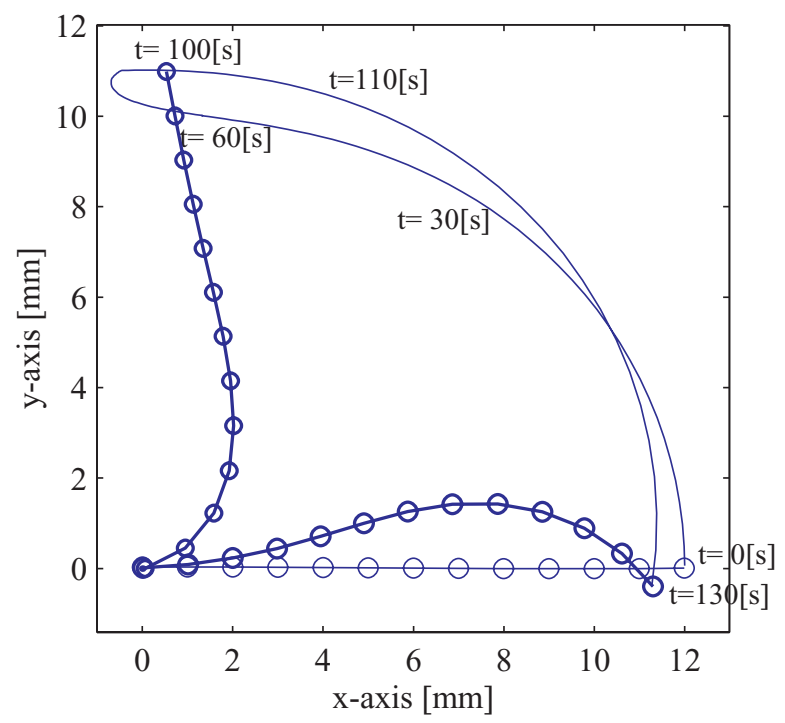

Figure 7: Macro position control by large deformation

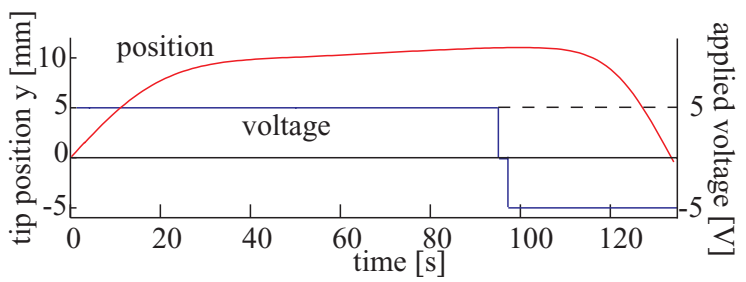

Figure 8: Applied voltage and tip position of y

\subsection{Dynamic change of configuration of the gel}

First of all, we describe the way of changing the path of the gel manipulator dynamically. If we keep on applying the electric field, the gel manipulator bends towards the electrodes and goes along the electric field. Once the orientation of the link is perpendicular toward the electrode, the diffusion speed exceeds the adsorption speed of molecules. This make the joint angles of the tip to becomes larger compared to those of the root, which make the $\mathrm{x}$-coordinate of the tip becomes larger while $\mathrm{y}$ coordinate of the tip remains constant (Figure 7, $\mathrm{t}=60$ to $100[\mathrm{~s}]$ ). After keeping the $\mathrm{y}$-coordinate of the tip toward one side, we apply $-5[\mathrm{~V}]$ until the tip goes back to the original position (Figure 7, $\mathrm{t}=130[\mathrm{~s}]$ ). The gel becomes convex shaped which make the x-coordinate of the tip becomes smaller, $\mathrm{x}=11.3[\mathrm{~mm}]$. Path of the tip and initial $(\mathrm{t}=0[\mathrm{~s}])$, transitional $(\mathrm{t}=100[\mathrm{~s}])$ and final $(\mathrm{t}=130[\mathrm{~s}])$ shape of the gel are illustrated in Figure 7. The applied voltage and the y-coordinate of the tip is shown in Figure 8 . The initial applied voltage was $5[\mathrm{~V}]$ until the tip reached $\mathrm{y}=11[\mathrm{~mm}]$, and its polarity was reversed to $-5[\mathrm{~V}]$ until the tip reached $\mathrm{y}=0[\mathrm{~mm}]$.

In this way, we can move the path of the tip by dy-

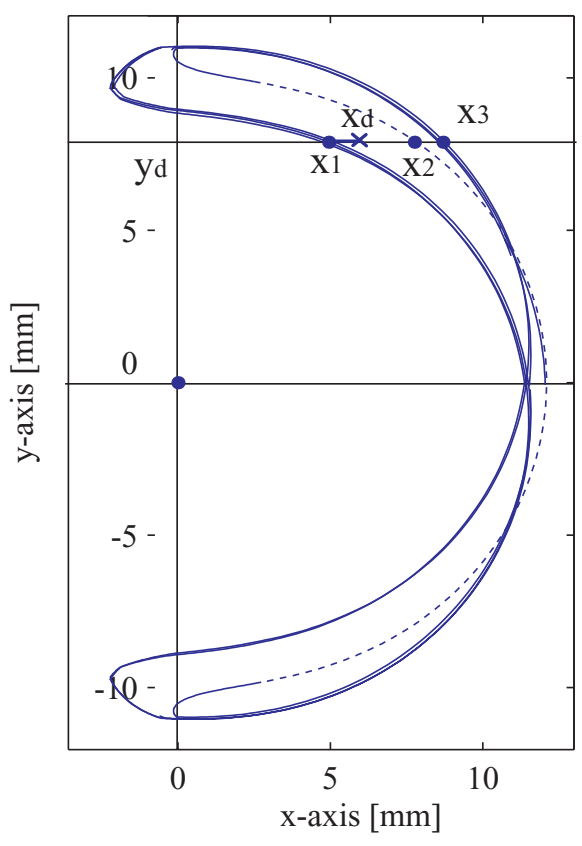

Figure 9: Path of the gel manipulator

namically changing the configuration of the gel manipulator based on deformation mechanism. Figure 9 show the path of the tip which moves between $10[\mathrm{~mm}]$ to $10[\mathrm{~mm}]$ (dotted line), and $11[\mathrm{~mm}]$ to $-11[\mathrm{~mm}]$ (continuous line). The tip moves on the dotted line for the first time and transit to the continuous line. But still, the $\mathrm{x}$ and y-coordinate of the gel is coupled although the combination is different from the original ones. We would like to slightly change the $\mathrm{x}$-coordinate of the tip while maintaining the y-coordinate of the tip. We will describe the method in the next subsection.

\subsection{Slight change of configuration of the gel}

Now that we can change the path dynamically, we would like to adjust the position. By applying oscillating electric field with small amplitude, we can slightly change the configuration.

The basic idea is that we need to curve or straighten the overall shape of the gel to adjust tip position. The $\mathrm{x}$-coordinate of the gel at the same $\mathrm{y}$-coordinate becomes relatively smaller if the gel manipulator curves. The method to straighten the curved gel is:

- Move the tip of the gel by the electric field to the center of the original position so that the orientation of the gel becomes vertical to the electric field.

- Apply oscillatory electric field at that position.

- The gel straighten because the molecules adsorb and desorb uniformly along the longitude of the gel. 


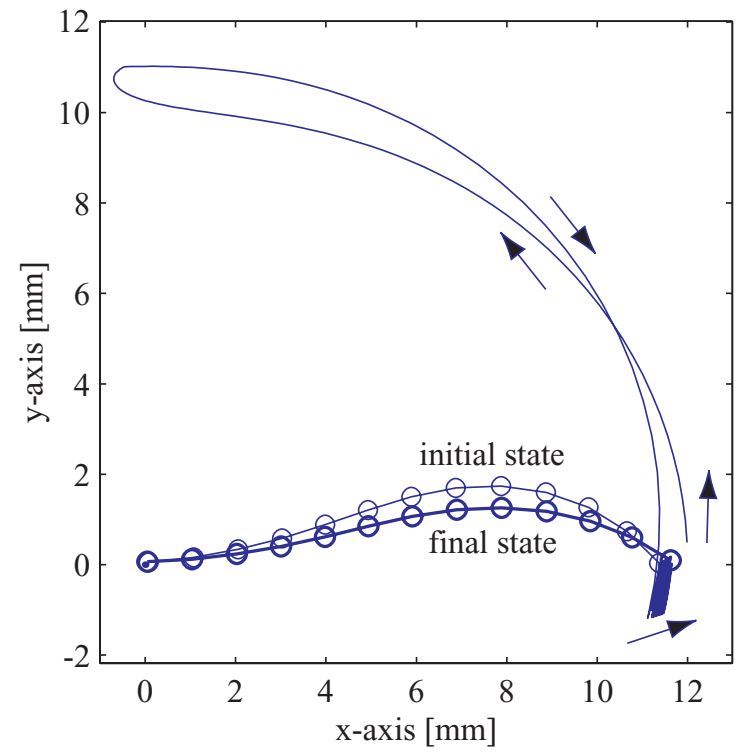

Figure 10: Micro position adjustment by oscillation

The method to curve the straight gel is:

- Move the tip of the gel by the electric field to the anode electrode so that the orientation of the gel becomes parallel with the electric field.

- Apply oscillatory electric field at that position.

- The mechanism of slight change is almost the same as dynamic change, the desorption of molecules near the tip.

We will show examples respectively. For the first case, we move the tip of the gel to $y=11[\mathrm{~mm}]$ and move back to $\mathrm{y}=0[\mathrm{~mm}]$. Then apply $-5[\mathrm{~V}]$ and $5[\mathrm{~V}]$ repeatedly for 20 times so that the gel oscillates between $y=-1[\mathrm{~mm}]$ and $0[\mathrm{~mm}]$. The initial shape and final shape, and the path of the gel are shown in Figure 10. The tip position is (11.6, 0 ) whose $\mathrm{x}$-coordinate became larger. For another case, we start with straight one. Move the tip to $\mathrm{y}=8[\mathrm{~mm}]$ and oscillates between $\mathrm{y}=8[\mathrm{~mm}]$ and $9[\mathrm{~mm}]$ for 20 times and move back to $0[\mathrm{~mm}]$. The results are shown in Figure 11. The tip position is $(11.9,0)$. The gel deforms into convex shaped, whose joint angles are smaller compared to the one which is illustrated in Figure 7.

\subsection{Selection of the path to reach the desired po- sition}

There are three ways to reach the tip to the desired position $\left(x_{d}, y_{d}\right)$. The cross section of the path and the line $y=y_{d}$ is $\boldsymbol{r}_{\boldsymbol{i}}=\left(x_{i}, y_{d}\right)(i=1,2,3)$ (Figure 9). We need to select one of the position and adjust the gel to the desired position. We name the controller function $\boldsymbol{u}_{\boldsymbol{i}}(i=1,2,3)$ which corresponds to $\boldsymbol{r}_{\boldsymbol{i}}(i=1,2,3)$. Then

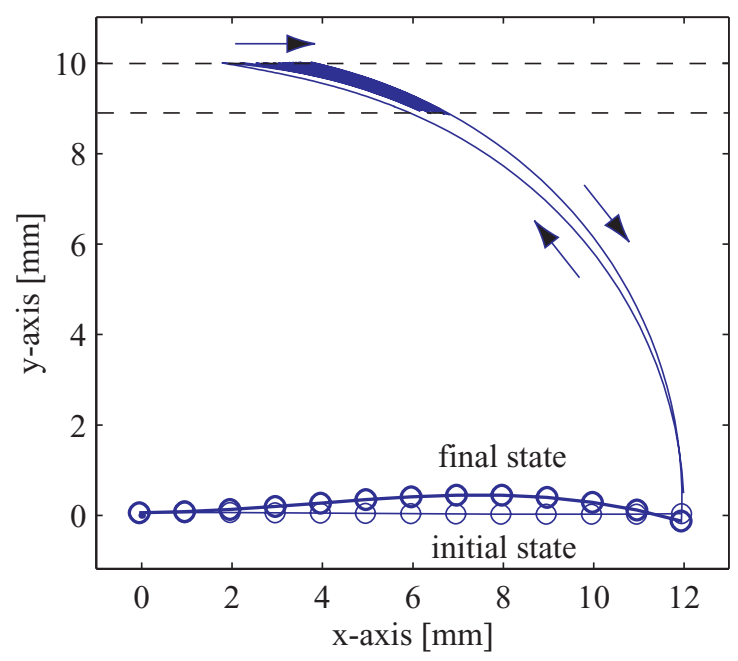

Figure 11: Micro position adjustment by oscillation II

we adjust the tip by oscillatory motion. In all cases, the controller estimate the $\mathrm{x}$-coordinate at $y=y_{d}$ in each period of oscillation and if the condition is satisfied, oscillation will be stopped and the tip will move to the goal.

We use total time to reach the goal for cost. We name the cost of controller function $\boldsymbol{u}_{\boldsymbol{i}}(i=1,2,3)$ for $J_{i}(i=$ $1,2,3)$ and calculate

$$
J_{\min }=\min \left[J_{1}, J_{2}, J_{3}\right]
$$

We select controller function $\boldsymbol{u}_{\boldsymbol{i}}$ that corresponds to $J_{\text {min }}$.

\subsection{Simulation and Experimental Results}

We performed experiments to examine the method. We show the simulation and experimental results of the final shape and path to reach the goal position $(9.5,6.0)$. We calculated the criterion function $J_{\min }=J_{1}=416$. The path of the tip and final shape of the simulation are illustrated in Figure 12, and final shape real gel are shown in Figure 13. Repeated time of oscillation is 10 times.

\section{Conclusions}

In this paper, we showed a method to solve inverse kinematics of gel robots made of electro-active polymer gel. As a first step, we proposed a method to control the tip position of a manipulator entirely made of electro-active polymer gel. We made clear that the various shapes of the gels can be derived by applying time alternating electric fields even if those are spatially uniform.

The problem is we cannot control the gel manipulator directly, because the electro-active polymer gels which we use in this paper are driven by separated electrodes and their motions are coupled. We examined the constitutive equation and proposed the method of changing the 


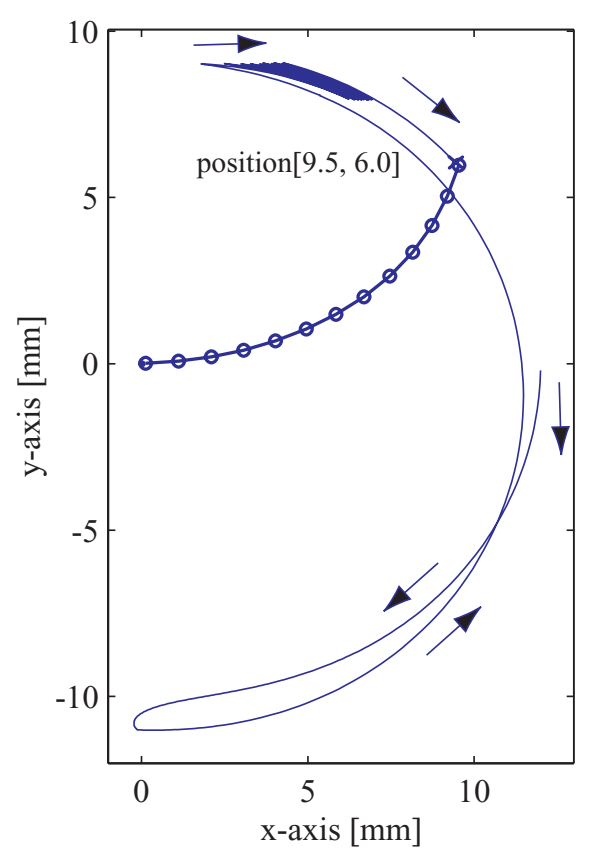

Figure 12: Path of the tip and final shape of the gel

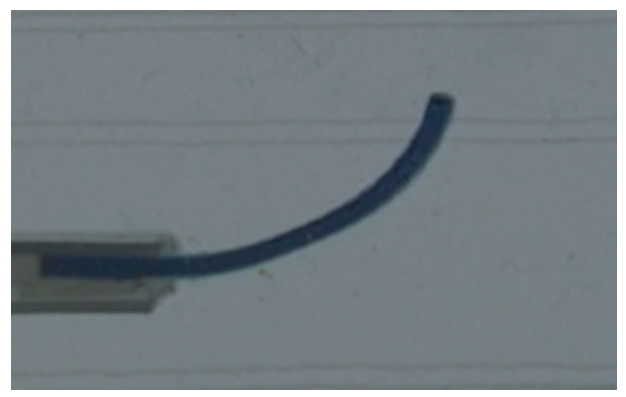

Figure 13: The gel manipulator which reached the final goal

configuration of joint angles by altering the orientation of each link to the spatially uniform electric field. The interesting point is that the tip of the gel manipulator can reach finite area in the electric field, not only on the narrow arc.

First, we demonstrated the dynamic change of configuration of the gel manipulator when almost all links goes with the electric field. Then, we presented the slight change of configuration with oscillating electric field. Finally, by combining the above methods which generate macro-micro motion, we controlled the tip position of the gel manipulator.

Our method generates subset of possible workspace and moves the tip of the gel in the plane. From the viewpoint of problem solving algorithm, we can evaluate our inverse kinematic model as practical approach; efficiency is in high priority and optimality and completeness are ne- glected. This research will be one of the fundamental example to control deformable robot made of electro-active polymer gel. Future works will include exploring more optimal and complete algorithm. We would like to sophisticate the method by thorough simulation and apply them to the shape control of real gel robots.

\section{Acknowledgments}

This work is supported by the Japan Society for the Promotion of Science Grant for Research For The Future JSPS-RFTF96P00801. The authors express deep appreciation to Prof. Y. Kakazu, Prof. Y. Osada of Hokkaido University who give us suggestions of our research.

\section{References}

[1] R. Pelrine and R. Kornbluh and Q. Pei and J. Joseph, "High-Speed Electrically Actuated Elastomers with Strain Greater Than 100\%," Science, vol. 287, pp. 836-839, 2000.

[2] E. W. H. Jager, O. Inganäs, and I. Lundström, "Microrobots for Micrometer-Size Objects in Aqueous Media: Potential Tools for Single-Cell Manipulation," Science, vol. 288, pp. 2335-2338, 2000.

[3] S. Sewa, K. Onishi, K. Asaka, N. Fujiwara, and K. Oguro, "Polymer Actuator Driven by Ion Current at Low Voltege, Applied to Catheter System," in Proc. MEMS 98, 1998, pp. $148-153$.

[4] M. Konyo, S. Tadokoro, and T. Takamori, "Artificial Tactile Feel Display Using Soft Gel Actuators," in Proceedings of the 2000 IEEE International Conference on Robotics and Automation, 2000, pp. 3416-3421.

[5] M. Otake, Y. Kagami, M. Inaba, and H. Inoue, "Behavior of a Mollusk-Type Robot Made of Electro-Active Polymer Gel under Spatially Varying Electric Fields ," in Intelligent Autonomous Systems 6 E.Pagello et al.(Eds), 2000, pp. 686691.

[6] M. Otake, M. Inaba, and H. Inoue, "Development of Gel Robots made of Electro-Active Polymer PAMPS Gel," in Proceedings of the 1999 IEEE International Conference on Systems Man and Cybernetics, 1999, vol. II, pp. 788-793.

[7] M. Otake, M. Inaba, and H. Inoue, "Kinematics of Gel Robots made of Electro-Active Polymer PAMPS Gel," in Proceedings of the 2000 IEEE International Conference on Robotics and Automation, 2000, pp. 488-493.

[8] M. Otake, Y. Kagami, M. Inaba, and H. Inoue, "Dynamics of Gel Robots made of Electro-Active Polymer Gel," in Proceedings of the 2001 IEEE International Conference on Robotics and Automation, 2001, pp. 1458-1462.

[9] Y. Osada, H. Okuzaki, and H. Hori, "A Polymer Gel with Electrically Driven Motility," Nature, vol. 355, pp. 242244, 1992.

[10] Y. Osada, H. Okuzaki, J.P. Gong, and T. Nitta, "Electro-Driven Gel Motility on the Base of Cooperative Molecular Assembly Reaction," Polym. Sci., vol. 36, pp. 340351, 1994.

[11] H.Okuzaki and Y. Osada, "Effects of Hydrophobic Interaction on the Cooperative Binding of Surfactant to a Polymer Network," Macromolecules, vol. 27, pp. 502-506, 1994. 